\title{
Evolução das Inteligências Múltiplas pelo Método da Espiral de Aprendizagem de Kolb utilizando PSO
}

\author{
Fábio F. de Moura ${ }^{1}$, Lucas M. Franco ${ }^{1}$, Sara L. de Melo ${ }^{1}$, Márcia A. Fernandes ${ }^{1}$ \\ ${ }^{1}$ Departamento de Ciência da Computação \\ Universidade Federal de Ubelândia \\ Uberlândia, Minas Gerais, Brasil \\ \{fabioferreirademoura, lmarchesoti, saraluziamelo\}@gmail, marcia@ufu.br
}

\begin{abstract}
The learning and the pursuit of knowledge is a paradigm that comes with being human. Increasingly notices the computer filling out this scenario with the inclusion of new technologies that provide alternative ways of aggregating the inclusion and propose to teaching and learning. This paper describes a simulator that uses a Particle Swarm Optimization technique to suggest the provision of learning objects based on the Theory of Multiple Intelligences and Learning Spiral. In addition, certain stimulating multiple intelligences through student teaching method proposed by Kolb. The concepts used here can be used in virtual learning environments.
\end{abstract}

\begin{abstract}
Resumo. O aprendizado e a busca de saberes é um paradigma que acompanha o ser humano. Cada vez mais nota-se a informática preenchendo este cenário com a inserção de novas tecnologias que propiciam formas alternativas de agregar a inclusão e de propor caminhos que auxiliem no processo de ensino $e$ aprendizado. Este artigo descreve um simulador que utiliza a técnica Particle Swarm Optimization para sugerir Objetos de Aprendizagem baseado na Teoria das Inteligências Múltiplas e a Espiral de Aprendizagem. Além disso, estimular determinadas inteligências múltiplas do estudante através do método de ensino proposto por Kolb. Os conceitos aqui empregados poderão ser utilizados em ambientes virtuais de aprendizagem.
\end{abstract}

\section{Introdução}

Em sala de aula convencional ou em ambiente virtual de aprendizagem alguns fatores que aparecem e que podem ser obstáculos para o aprendizado são as técnicas empregadas e a forma como é apresentado o conteúdo ao qual pode estimular os saberes no estudante. Pesquisas mostram que muito se tem construído nesse universo para promover a adaptação e a formatação de conteúdo, além de sistemas completos que promovem adaptatividade e adaptabilidade ao usuário.

Teorias educacionais têm sido utilizadas no sentido de acompanhar e promover o ensino em cursos via computador. Através destas teorias é possível extrair características para oferecer um conteúdo personalizado. Muitas ferramentas já foram construídas, inclusive sistemas completos que promovem adaptatividade e adaptabilidade ao usuário, como um Sistema Hipermídia Adaptativo para seleção de conteúdos, ou uma metodologia para construir tais sistemas. 
A maioria destes sistemas são amparados, pedagogicamente, por alguma teoria de estilos de aprendizagem para oferecer material didático adequado e personalizado ao estudante. Assim, o material é oferecido ao estudante de acordo com as suas características particularizadas. Entretanto, o processo de aprendizagem, ainda que considerando o perfil do estudante e estilos de aprendizagem, não é direcionado, pois não identifica os diferentes estágios para aprendizagem de um conteúdo.

Neste trabalho, é proposto um modelo que permite adaptatividade, utilizando as teorias de Kolb para guiar o processo de aprendizagem e também a teoria de Inteligências Múltiplas para auxiliar na seleção de Objetos de Aprendizagem (OA) mais adequados em cada módulo do curso. Além disso, promove a evolução do estudante em outros aspectos de sua forma de aprender. Desta maneira, pretende-se estimular as Inteligências Múltiplas (IM) de um estudante através de sua evolução pela espiral de aprendizagem.

Para descrever o modelo computacional proposto, o artigo está organizado em cinco seções. Sendo que, a segunda seção mostra conceitos fundamentais utilizados no trabalho. Na terceira seção são mostrados trabalhos correlatos que usam Sistemas Adaptativos, bem como a técnica Particle Swarm Optimization (PSO) utilizada para modelar a otimização do sistema . Na quarta seção é descrito a evolução do estudante através das teorias educacionais utilizadas. Na seção cinco são mostrados os resultados. Finalmente, na seção seis tem-se a conclusão.

\section{A Espiral de Aprendizagem de Kolb e as Inteligências Múltiplas}

Estilos de Aprendizagem (EA) definem maneiras às quais a mente de uma pessoa utiliza para aprender algo que lhe é proposto. Tais estilos são únicos e pessoais, pois cada indivíduo apresenta facilidade com um determinado estilo e dificuldade em outros [Borges 2011]. Existem diversas teorias (mais de sessenta [Barros 2011]) que definem de forma alternativa os EA's, dentre essas teorias destaca-se a Espiral de Aprendizagem de Kolb, que origina os EA's de Kolb. Outra teoria que é bem utilizada no ramo da psicopedagogia são as Inteligências Múltiplas (IM) [Gardner 1994] e que em muitas vezes são confundidas com EA [Prashnig 2005]. As IM's indicam um quantitativo de inteligência para algumas áreas do conhecimento (i.e. inteligência ligada à linguagem em palavras - Inteligência Linguista). [Gardner 1994] identificou oito inteligências independentes e cogita-se a existência de mais algumas. As IM's servem para inferir o grau de afinidade que um estudante têm com determinada área do conhecimento, enquanto os EA's definem o formato como ele gosta de visualizar o que lhe está sendo proposto.

A teoria da aprendizagem experiencial de Kolb [Kolb 1999]descreve quatro dimensões de desenvolvimento: estrutura afetiva (Experiência Concreta -EC), estrutura perceptual (Observação Reflexiva - OR), estrutura simbólica (Conceituação Abstrata CA) e estrutura comportamental (Experimentação Ativa - EA) [Cerqueira 2000], conforme ciclo quadrifásico mostrado na Figura 1 (a). Estas dimensões ao serem combinadas, duas a duas, em quadrantes, definem EA, podendo ser vistos como "áreas" triangulares, conforme Figura 1 (b).

O Inventário de Estilos de Aprendizagem (IEA) [Almeida et al. 2011], composto de doze sentenças que definem o tamanho de cada dimensão e, por conseguinte, a área de cada quadrante e mostra o grau de cada EA. Os EA's de Kolb formados entre as quatro dimensões são: divergentes (concreto, reflexivo) que são estudantes que respondem bem 


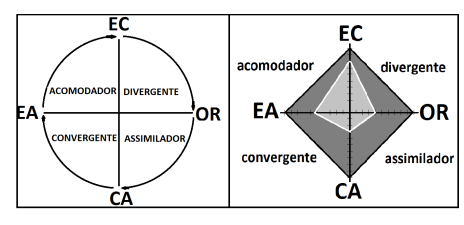

(a)

(b)

Figura 1. Ciclo Quadrifásico de Kolb.

a explicações de como o material estudado se refere a suas experiências; assimiladores (abstrato, reflexivo) que são estudantes que aprendem melhor com informações apresentadas de maneira organizada, lógica, e obtêm aproveitamento melhor se tiverem tempo para refletir; convergentes (abstrato, ativo) são estudantes que gostam de ter a oportunidade de trabalhar ativamente em tarefas bem definidas, e de aprender por tentativa e erro; acomodadores (concreto, ativo) são estudantes que aplicam as informações em novas situações para resolver problemas reais [Cerqueira 2000].

Ao detectar o EA que um indivíduo possui, pode-se desenvolver uma metodologia de ensino adaptada a ele para que o mesmo entenda o que está sendo proposto de maneira mais compreensível ao seu estilo, ou então estimular estilos não predominantes de seu perfil. Para verificar se houve aprendizado, além de observar a maximização da área Figura 1 (b). [Gardner 1994] afirma que o desenvolvimento cognitivo é a capacidade de entender e expressar significado em vários sistemas simbólicos utilizados num contexto cultural. Em sua teoria Gardner afirma que existem formas independentes de percepção, memória e aprendizado. Para Gardner o ser humano possui várias inteligências sendo algumas, maior ou menor, em determinadas áreas de atuação. Gardner faz uma abolição à ideia de Quociente de Inteligência (QI) que engessa de forma unitária a inteligência do indivíduo. Existem nove inteligências descritas por [Gardner 1994].

Neste trabalho são utilizadas quatro IM (Linguístico-verbal (LV), Lógicomatemática (LM), Visual-espacial (VE) e Cinestésico-corporal (CC)), pois, conforme [Barbosa 2004], é possível associá-las a diferentes mídias, pensando-se numa futura construção de Objetos de Aprendizagem (OA) - material de estudo. [Barbosa 2004] salienta-se que esta "simplificação" não traz prejuízos posto que as IM's são independentes entre si e não há evidências de que os indivíduos possuam um valor de IM que deve ser a totalização de todas as IM, que implicaria em perdas caso algumas não fossem consideradas.

No desejo de mostrar que pode-se utilizar mais de uma teoria de aprendizagem para auxiliar na escolha de material de estudo, ou OA, e "medir" o aprendizado adquirido pelo estudante, este trabalho utiliza a Espiral de Aprendizagem de Kolb para formatação pedagógica de um curso passando pelas quatro fases da espiral e, com a teoria das IM's, deseja-se inferir o quanto um estudante melhorou o nível de cada uma de suas inteligências após passar pelo processo de ensino proposto por Kolb. Neste sentido criou-se um modelo computacional que simula um curso via computador, em que um estudante tem mapeado suas IM, bem como as dimensões da espiral de Kolb. Neste modelo o algoritmo do PSO é usado para inferir dados sobre este estudante e sobre uma turma que o acompanha durante o processo de aprendizado. 


\section{Sistemas Adaptativos via Computador e PSO}

Sistemas Adaptativos Educacionais cada vez mais fazem parte do contexto de pesquisa em informática na educação onde a computação juntamente com a Inteligência Artificial (IA) trazem benefícios e resultados progressivos para o aprendizado de estudantes tanto no contexto de e-learning quanto presencial, fornecendo a adaptatividade ou adaptabilidade.

Promover adaptatividade em sistemas educacionais é quando se extrai características particulares de um estudante por um sistema à parte e, logo após, retorna tais informações a um Learning Management System (LMS), um exemplo de adaptatividade é fazer com que um LMS execute a metodologia de ensino mais adequada para um determinado estudante ou um grupo deles. Dessa maneira, promove-se uma melhoria considerável no ensino, já que se estaria abordando fatores particularizados do estudante. Pode-se também promover adaptabilidade que é a capacidade do sistema em permitir ao usuário alterar certos parâmetros relativos às funcionalidades do sistema, modificando a forma de acesso aos recursos [Mota 2010] [Moura and Fernandes 2012].

Existem sistemas que proporcionam, de forma eficaz, o processo educacional à distância com adaptatividade e adaptabilidade. [Melo 2003] propõe um modelo, baseado em computador, para auxiliar na detecção precoce das IM's. [Barbosa 2004] propõe uma metodologia para construir sistemas que realizem adaptação automática, na interface de uma hipermídia, conforme o perfil do usuário usando também a Teoria das IM's. [Puga 2008] propõe um Sistema Hipermídia Adaptativo, cujos critérios para adaptação consistem na seleção de conteúdos. [Dorça et al. 011a, Dorça et al. 011b] propõe a combinação de EA e na correção dinâmica das inconsistências no Modelo do Estudante (ME), levando em consideração o forte aspecto não-determinístico do processo de aprendizagem. [Menolli and et al 2011] propõe uma abordagem para gerar objetos de aprendizagem a partir de páginas wiki, utilizando tecnologias semânticas e o padrão Learning Object Metadata (LOM), a fim de que a informação possa ser organizada de modo que possa ser melhor reutilizada, melhorando assim a aprendizagem social. [Valaski and et al 2011] efetuou uma revisão do modelo de Felder e Silverman como o mais utilizado para a identificação do estilo de aprendizagem seguido do modelo de Kolb.

Observando-se pesquisas correlatas nota-se que existe uma carência em relacionar mais de uma teoria pedagógica no processo de adaptatividade. Nesse intuito, para uma escolha mais precisa de material de estudo surge a ideia de analisar as características individuais do estudante e fazer com que este passe pelo processo pedagógico da Espiral de Aprendizagem de Kolb e, no final do processo, utiliza-se como parâmetro para medir o aprendizado o nível de ganho em suas IM's. Para modelar este processo usou-se o PSO. O Particle Swarm Optimization (PSO) [Kennedy and Eberhart 1995] constitui-se numa técnica da inteligência coletiva baseada em uma população de soluções e transições aleatórias que apresenta características similares às técnicas da computação evolutiva, e é motivado pela simulação de comportamento social e cooperação entre agentes [Tebaldi et al. 2006].

\section{Estimulando IM através da Espiral de Aprendizagem}

Baseado pedagogicamente pelos conceitos apresentados, foi criado um simulador denominado Sistema Adaptativo de Ensino que usa PSO - SAEP, amparado pela teorias das IM e nos EA de Kolb. No SAEP as dimensões da Espiral de Aprendizagem de Kolb e 
as IM's foram consideradas a "entrada" do processo de aprendizagem. Após inferir uma metodologia baseada tanto pela espiral quanto pelas IM's, a "saída" desse processo mostra o grau de afinidades que o individuo possui com o conteúdo e a forma que este lhe foi mostrado, "medindo" o quanto o estudante adquire de conhecimento com o estímulo de suas inteligências quanto no avanço na espiral de aprendizagem.

Para o SAEP o estudante responde ao IEA e às sentenças de Gardner, para inferir seus níveis nas dimensões de Kolb e de IM's. Computacionalmente o estudante é representado por um vetor que contém 52 posições, sendo que as 48 primeiras são referentes às dimensões de Kolb (as 12 primeiras EC, as 12 próximas OR, depois CA e as 12 últimas referentes à EA). As quatro últimas posições são referentes ao percentual das quatro IM. Após detectadas as dimensões e as IM's, observa-se na espiral qual estilo de aprendizagem é mais dominante no estudante e inicia-se o processo de giro. Sendo que para isso o objetivo é otimizar o nível de EA e de IM do estudante.

Para essa otimização necessita-se conhecer o que seria um nível "máximo" de cada IM nos quadrantes da espiral. Sendo assim, inicialmente foi feita uma pesquisa com quatorze profissionais da educação, sendo eles, pedagogos e psicopedagogos. Essa pesquisa consistiu em analisar o IEA de Kolb e respondê-lo de uma maneira diferenciada por esses profissionais. Nessa etapa foram atribuídos pesos (de um a quatro) nas sentenças do IEA e, posteriormente respondido de acordo com as características de estudantes que fossem extremamente ligados àquela determinada IM, e por cada estrutura de aprendizagem de Kolb.

Esse questionário foi respondido quatro vezes, para cada vez, foi associada uma IM estudada no referente artigo. Com isso pode-se obter em Kolb uma ideia do que seria um perfil ideal, entre as quatro dimensões, para as quatro IM's, obtendo quatro "perfis" ótimos para cada EA de KOlb (LV - LM - VE - CC). Nesse sentido, fez-se uma média para as respostas adquiridas fazendo-se a modelagem computacional dos pontos ótimos. A modelagem dos ótimos é dada por um vetor de 30 posições, que recebe valores da seguinte maneira: as posições ímpares de um a vinte e quatro recebem os valores referentes à primeira dimensão, onde o estudante passa pela espiral de Kolb e as posições pares recebem os valores referentes à segunda dimensão, sendo que é atribuído o valor 1 para quando essa média for maior e 0 quando menor; a posição vinte e cinco recebe o somatório das posições ímpares; a posição vinte e seis recebe o somatório das posições pares; as posições de vinte e sete a trinta recebem o percentual das IM (LV, LM, VE e CC, respectivamente).

Para o simulador, que usa a ideia do PSO, coloca-se um estudante a ser observado como primeira posição de uma matriz e $n$ estudantes modelados como um enxame de partículas do PSO, onde cada estudante é uma partícula e participa da evolução. O PSO é utilizado para fazer uma predição de qual será a melhor sugestão de material - OA a ser oferecido levando-se em consideração os ótimos e as características dos próprios estudantes. O Algoritmo 1 mostra a ideia geral do simulador.

No Algoritmo 1, conforme descrito, o estudante responde ao IEA de Kolb e ao questionário de Gardner, o que foi reduzido por [Barbosa 2004], começando o processo de aprendizado. Inicialmente, verifica-se em que ponto da espiral o estudante possui maior afinidade - maior área - e, o primeiro giro parte desse ponto e parte-se do pressuposto que 


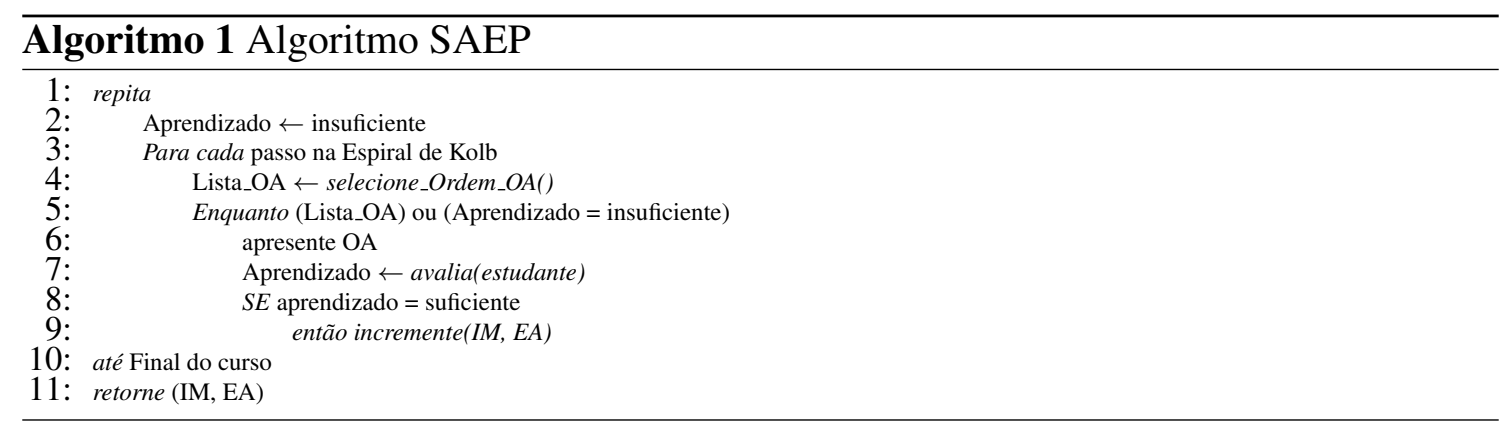

este estudante é "reprovado". Leva-se para a espiral as dimensões atuais do estudante para o PSO onde este recomenda uma ordem de sugestões de tipos de OA's a serem mostrados ao estudante. Se o OA for satisfatório (estudante $\leftarrow$ aprovado) então o estudante parte para um próximo ponto na espiral (mais um módulo do curso), até que o curso (seus módulos) sejam finalizados.

No PSO as partículas são representadas pelas dimensões de Kolb referentes ao ponto na espiral, considerando a distância euclidiana entre o estudante e os ótimos. Para representar o efeito das IM's, ponderou-se a distância de Kolb com a IM da partícula referente a cada ótimo. Dessa forma a distância varia para indicar facilidade de aprendizado dependendo do valor da IM. A fórmula utilizada foi $\mathrm{dist}_{k o l b} / \mathrm{gard}_{o a}$ e produz um resultado entre 0 e infinito. A velocidade segue a fórmula padrão do PSO, onde as distâncias entre a partícula e os ótimos são calculadas como sendo apenas a distância das dimensões de Kolb. Não consideramos Gardner neste momento pois a partícula se move apenas no espaço de Kolb, então não faz sentido acelerá-la com uma força externa. As partículas são guiadas pelos ótimos e buscam se posicionar em cada um deles, porém como se baseiam em mais de um ótimo ao mesmo tempo e também são influenciados por características individuais $\left(p_{\text {best }}\right)$, os estudantes tendem a estabilizar em uma posição que leva em conta todos estes fatores. O efeito do $g_{\text {best }}$ foi suprimido ao longo das iterações para auxiliar a estabilização. Desta forma ele age como um auxílio no início da execução e deixa que cada partícula se estabilize considerando apenas suas características individuais.

A recomendação é baseada na distância do estudante a cada ótimo. Assumindo que a partícula atinge o ponto estável, recomenda-se OA em ordem crescente de distância até que o estudante aprenda ou esgote os OA's. Em seguida o estudante continua o giro na espiral. Como o aprendizado esta ligado à IM, considerou-se como probabilidade de aprendizado a IM relativa ao OA.

\section{Resultados e Discussão}

Os resultados foram analisados em um primeiro momento de forma visual, baseado na metodologia de [Fatahi and Ghasem 2010], onde foram consideradas a distância que o estudante encontra-se dos OA, a nota recebida ao efetuar quatro questões ao final do módulo e o tempo que o estudante leva para fazer cada questão, sendo que os parâmetros foram escolhidos por quatorze pedagogos. Em relação a estes resultados, pode-se observar que, em um exemplo, para um estudante que possui o seguinte "perfil" inicial na espiral: $E C=2, O R=5, C A=2$ e $E A=3$. Após quatro ciclos completos (dezesseis módulos do conteúdo) foram obtidos os seguintes valores de dimensões: $E C=8.5$, $O R=10.2, C A=5.4$ e $E A=3.9$ (as dimensões neste teste não foram balanceadas no 
intervalo $[0,1]$. Assim, o estilo divergente do estudante iniciou com área igual a 5 e finalizou com área igual a 43.35; o estilo assimilador iniciou com área igual a 5 e finalizou com área igual a 27.54; o estilo convergente iniciou com área igual a 3 e finalizou com área igual a 10.53 e o estilo acomodador iniciou com área igual a 3 e finalizou com área igual a 16.6. Em relação às IM's, a inteligência LV passou de 0,625 a 0,660, a LM de 0,695 a 0,706, a VE de 0,625 a 0,628 e, por último, a CC permaneceu constante em 0, 750.

Foi efetuada uma recomendação baseada nas IM's que o estudante possui, com quatro tipos de oferecimentos de OA (Recomendação aleatória: onde faz-se uma roleta e sorteia-se a ordem dos OA; Recomendação baseada nas IM's: onde se dá preferência ao oferecimento de OA baseado no nível de IM que o estudante possui, oferecendo OA em ordem decrescente ao seu nível de IM; Recomendação pelo PSO clássico [Kennedy and Eberhart 1995], que escolhe qual OA possui menor distância euclidiana em relação aos demais; e, Recomendação baseada numa modificação do $g_{\text {best }}$ durante as iterações do PSO, para averiguar como o estudante evolui com um pouco mais de independência em relação à turma. Na versão modificada do PSO, o efeito do $g_{b e s t}$ na velocidade da partícula é ponderado de acordo com a Equação 1.

$$
C_{2}=C_{2_{0}} *\left(1-\frac{k}{k_{\max }}\right)
$$

onde $C_{2_{0}}$, é o valor inicial de $C_{2}$, nesse caso $\left(C_{2}=2\right), k$ representa a iteração corrente do PSO e $k_{\max }$ representa o número máximo de iterações do PSO.

Para os testes com a "avaliação baseada em Gardner (IM)" foram usados alguns parâmetros do algoritmo que são:

1. $V_{0}=0,01$ (velocidade inicial para o algoritmo clássico do PSO e no modificado);

2. $c_{1}=2$ (fatores cognitivos individual);

3. $c_{2_{i}}=2$ (fatores cognitivos social);

4. $w_{\min }=0,4$ (o mínimo da inércia);

5. $w_{\max }=0,9$ (o máximo da inércia);

6. iter $_{\max }=1000$ (o número máximo de iterações do PSO e do Pso Modificado).

Outros valores foram testados para tais parâmetros, mas o observado foi um comportamento similar e estes são recomendados por [Kennedy and Eberhart 1995, Shi and Eberhart 1998, Bansal et al. 2011].

Como exemplo de resultado tem-se a Tabela 1 que mostra tipos de recomendação o estudante atinge êxito em melhoria tanto na Espiral de Aprendizagem, quanto em seus padrões de inteligências. Isto é um fator positivo mostrando que um Sistema Adaptativo com recomendação de OA baseado em teorias cognitivas será melhor do que um sistema que ofereça apenas um tipo de $\mathrm{OA}$ a estudantes, pois estes não estariam estimulando fatores particularizados e melhorar aqueles que estão num nível abaixo. Na Tabela 1, o estudante possui EA divergente e IM linguista-verbal predominantes em relação aos demais. 


\section{Tabela 1. Comparativo Estudante com EA divergente e IM linguista-verbal predo- minantes em relação aos demais estilos e inteligências.}

\begin{tabular}{|l|l|l|l|l|l|l|l|l|}
\hline Processo Evolutivo & EC & OR & CA & EA & LV & LM & VE & CC \\
\hline \hline Perfil Inicial & 0.417 & 0.417 & 0.083 & 0.083 & 0.750 & 0.250 & 0.250 & 0.250 \\
\hline \hline Aleatório & 0.921 & 0.929 & 0.862 & 0.847 & 0.775 & 0.325 & 0.310 & 0.318 \\
\hline Melhora (\%) & 120,86 & 122,78 & 938,55 & 920,48 & 3,33 & 30,00 & 24,00 & 27,20 \\
\hline \hline Gardner & 0.929 & 0.929 & 0.876 & 0.876 & 0.775 & 0.325 & 0.318 & 0.325 \\
\hline Melhora (\%) & 122,78 & 122,78 & 955,42 & 955,42 & 3,33 & 30,00 & 27,20 & 30,00 \\
\hline \hline PSO & 0.921 & 0.892 & 0.830 & 0.876 & 0.772 & 0.302 & 0.318 & 0.325 \\
\hline Melhora (\%) & 120,86 & 113,91 & 900,00 & 955,42 & 2,93 & 20,80 & 27,20 & 30,00 \\
\hline \hline PSO modificado & 0.912 & 0.921 & 0.876 & 0.862 & 0.772 & 0.325 & 0.318 & 0.318 \\
\hline Melhora (\%) & 118,71 & 120,86 & 955,42 & 938,55 & 2,93 & 30,00 & 27,20 & 27,20 \\
\hline
\end{tabular}

Foram observados perfis de estudantes diferentes, tanto com predominância em IM's e EA's e os resultados foram satisfatórios. [Gardner 1995] relata que trabalhar com IM ainda é uma forma empírica de se avaliar estes níveis, o que foi feito em tais testes apresentados, mesmo que Gardner não seja contra as avaliações por uso de questionários. Por isso, optou-se em usar o questionário de [Antunes 001b], reduzido pelo estudo de [Barbosa 2004].

Os valores de acréscimo e de decréscimo para a evolução do estudante foram obtidos de forma experimental com a ajuda de pedagogos, que são profissionais que têm prioridade teórica para sugerir tais abordagens.

Um fato importante que foi observado é o de que o PSO modela o problema conceitualmente, já que a partir dele pode-se inferir as partículas como estudantes, os ótimos como objeto de aprendizagem (ou, o objetivo a ser alcançado pelo estudante) e a cooperação como sendo a "ajuda" que um estudante possa inferir noutro durante o processo de aprendizado, mostrando que o PSO varia de forma mais constante do que para os outros tipos de recomendação.

\section{Conclusões}

Considerando que cada pessoa possui uma forma particular de aprender. A promoção de adaptatividade em sistemas educacionais é uma alternativa na melhoria do ensinoaprendizado, já que se aborda fatores particularizados do estudante. Ao perceber que existem estudantes que aprendem de modo diferente, outras estratégias podem ser utilizadas para motivar esse estudante de forma mais eficaz.

Neste trabalho não objetivou-se a criação de um sistema adaptativo educacional completo. Foi proposto um modelo computacional utilizando Particle swarm optimization (PSO) - o SAEP - para a escolha da sugestão de objeto de aprendizagem a fim de auxiliar estudantes no processo de aprendizagem através do conhecimento de suas necessidades particulares através de sua evolução na Espiral de Aprendizagem de Kolb e, para confirmar o aprendizado é associada ao final do processo a relação da Espiral com a melhora do nível de IM do estudante. As partículas podem ser entendidas como um grupo de estudantes com características intrínsecas nos fatores presentes no questionário para detecção das dimensões de Kolb e no questionário para identificação das IM's.

Este trabalho mostrou que é possível associar as teorias de Kolb e de Gardner melhorando o ensino e aprendizado do estudante. Notou-se também que a contribuição 
de profissionais da área da educação é muito significativa e que técnicas de IA podem auxiliar na introdução de adaptatividade em sistemas educacionais.

Nas simulações executadas verificou-se que o uso do PSO para classificar OA satisfaz o processo de adaptatividade. Nota-se também que outras formas de adaptatividade, como a recomendação de OA baseado apenas nas inteligências múltiplas, podem também obter bons resultados, mas o fator cooperação do PSO é de grande relevância para obter um aprendizado contínuo, pensando-se que eles poderiam cooperar entre si num curso via computador, como nas salas de aula convencionais.

O uso do PSO mostrou-se interessante devido ao fato do espaço de busca para a otimização ser de dimensão grande ( $n=56$, inicialmente), que é a estrutura de cada partícula, ao combinar-se essa dimensão é gerado um espaço de busca com muitas possibilidades de respostas. Diante desta "explosão combinatória" o PSO mostra-se eficiente gerando resultados satisfatórios num curto intervalo de tempo.

\section{Referências}

Almeida, A. N. S., Machado, E. V., and Almeida, R. A. S. (2011). Estilos de aprendizagem de alunos surdos: uma análise cognitiva. V Colóquio Educação e Contemporaneidade, $3: 38-49$.

Antunes, C. (2001b). Como Identificar em Você e em Seus Alunos as Inteligências Múltiplas. Editora Vozes, Petrópolis, Brasil.

Bansal, J., Singh, P., Saraswat, M., Verma, A., Jadon, S., and Abraham, A. (2011). Inertia weight strategies in particle swarm optimization. Nature and Biologically Inspired Computing (NaBIC), 2011 Third World Congress on, pages 633-640.

Barbosa, A. T. R. (2004). Mecanismo para adaptação baseado em redes neurais artificiais para sistemas hipermidia adaptativos. In Tese de Doutorado. Universidade Federal de Santa Catarina.

Barros, D. M. V. (2011). Estilos de Aprendizagem na Atualidade: volume 1. Universidade Aberta de Portugal, Lisboa, Portugal.

Borges, F. N. (2011). Sistema tutor inteligente para o aperfeiçoamento da compreensão de leitura. XIV Simpósio Brasileiro de Informática na Educação - Anais do XXII SBIE - XVII WIE.

Cerqueira, T. C. S. (2000). Estilos de aprendizagem em universitários. In Tese de Doutorado. Universidade Estadual de Campinas, Faculdade de Educação.

Dorça, F. A., Lima, L. V., Fernandes, M. A., and Lopes, C. R. (2011a). Detecção e correção automática de estilos de aprendizagem em sistemas adaptativos para educação. RITA, 18(2):178 - 204.

Dorça, F. A., Lima, L. V., Fernandes, M. A., and Lopes, C. R. (2011b). A new approach to discover students learning styles in adaptive educational systems. XIV Simpósio Brasileiro de Informática na Educação, pages 70 - 79.

Fatahi, S. and Ghasem, N. (2010). Design and implementation of an intelligent educational model based on personality and learner's emotion. International Journal of Computer Science and Information Security, 7(3):1 - 13. 
Gardner, H. (1983). Frames of mind: The theory of multiple intelligences. New York: Basic Books Inc.

Gardner, H. (1994). Estrutura da mente: A Teoria das Inteligências Múltiplas. Editora Artes Medidas Sul LTDA, Porto Alegre, Brasil.

Gardner, H. (1995). Reflections on multiple intelligences: Miths and messages. Phi Delta Kappan, 77(3):200 - 209.

Kennedy, J. and Eberhart, R. (1995). Particle swarm optimization. In Neural Networks, 1995. Proceedings., IEEE International Conference on, volume 4, pages 1942-1948.

Kolb, D. A. (1999). The Kolb Learning Style Inventory. Version 3, Boston: Hay Group.

Kolb, D. A. and Kolb, A. Y. (2005). The kolb learning style inventory. In Manual Technical Specifications. Case Western Reserve University.

Melo, F. C. M. (2003). Modelo para auxiliar a detecção de inteligências múltiplas. Master's thesis, Universidade Federal de Santa Catarina. http://repositorio.ufsc.br/handle/123456789/86379?show=full.

Menolli, F. A. and et al (2011). Criação semi-automatica de objetos de aprendizagem a partir de conteúdos da wiki. XIV Simpósio Brasileiro de Informática na Educação, pages $70-79$. http://www.br-ie.org/pub/index.php/sbie/issue/view/48.

Mota, J. R. (2010). Adaptive-moodle: Adaptatividade e interoperabilidade em ambientes de e-learning utilizando tecnologias da web semântica. Master's thesis, Universidade Federal de Uberlândia. http://www.bdtd.ufu.br/.

Moura, F. F. and Fernandes, M. A. (2012). Uma proposta de um modelo computacional que usa $P S O$ para a escolha de objetos de aprendizagem baseado na espiral de kolb e nas inteligências múltiplas. XXIII Simpósio Brasileiro de Informática na Educação. http://www.br-ie.org/pub/index.php/sbie/article/view/1794/1555.

Prashnig, B. (2005). Learning styles vs. multiple intelligences (mi) two concepts for enhancing learning and teaching. Creative Learning Company. http://www.creativelearningcentre.com/downloads/.

Puga, A. G. (2008). Sistema hipermidia adaptativos para educação baseada na web: Uma visão semiótica. In Tese de Doutorado. Universidade de São Paulo.

Shi, Y. and Eberhart, R. (1998). A modified particle swarm optimizer. Proceedings of IEEE International Conference on Evolutionary Computation, pages 69-73. http://dsp.szu.edu.cn/pso/ispo/download/a

Tebaldi, A., COELHO, L. S., and LOPES, V. J. (2006). Detecção de falhas em estruturas inteligentes usando otimização por nuvem de partículas: fundamentos e estudo de casos. Sba Controle e Automação, 17(3).

Valaski, J. and et al (2011). Revisão dos modelos de estilos de aprendizagem aplicados a adaptação e personalização dos materiais de aprendizagem. XIV Simpósio Brasileiro de Informática na Educação, pages 844-847. 